\title{
Optical Control of Spatial Riemann Waves and Burgers' Equation Dynamics
}

\author{
Domenico Bongiovanni ${ }^{1,2}$, Benjamin Wetzel $^{3}$, Pengzhen Yang $^{2}$, Yi Hu$^{2}$, Yujie Qiu ${ }^{2}$, Jingjun Xü ${ }^{2}$, Stefan \\ Wabnitz $^{4}$, Zhigang Chen ${ }^{2,5}$, Roberto Morandotti ${ }^{1,6,7}$ \\ 1. INRS-EMT, 1650 Blvd. Lionel-Boulet, Varennes, QC J3X 1S2, Canada \\ 2. TEDA Applied Physics Institute and School of Physics, Nankai University, Tianjin 300457, China \\ 3. School of Mathematical and Physical Sciences, University of Sussex, Sussex House, Falmer, Brighton BN1 9RH, UK \\ 4. DIET, Sapienza University of Rome, Via Eudossiana 18, 00184 Rome, Italy \\ 5. Department of Physics \& Astronomy, San Francisco State University, San Francisco, CA 94132, USA \\ 6. Institute of Fundamental and Frontier Sciences, University of Electronic Science and Technology of China, Chengdu 610054, China
}

\section{ITMO University, St. Petersburg 197101, Russia}

Simple Riemann waves (RWs), solutions of the Inviscid Burgers' Equation (IBE), are of fundamental importance to study shock formation in different physical frameworks beyond hydrodynamics [1]. Recently, RW signatures in time domain have been reported in the context of nonlinear optical fibres [2-4]. Nevertheless, only limited control was demonstrated on the propagation of these peculiar optical pulses [5]. Here, we describe a method to control the nonlinear dynamics of their spatial counterpart, i.e., Riemann beams (RBs). Such RBs can be theoretically generated with arbitrary trajectories, by properly engineering an external potential and the application of an initial phase profile on the beam. In particular, we study shifted RBs, whose transversal shock position can be controlled, even in the absence of any external potential. Figures $1(a, b)$ illustrate the dynamical control achievable for two different cases of RBs. During propagation, a pre-chirped Gaussian beam maintains a constant peak intensity, and undergoes a progressive steepening of its trailing edge up to a near-vertical front at $\mathrm{z}$ $=10 \mathrm{~mm}$ (shock distance). Figure 1(a) shows a shifted Gaussian RB, generated by the inclusion of a linear phase shift $\alpha$. In Fig. 1(b), the external potential function and the initial phase are designed to guide the Gaussian RB along a sinusoidal path $\mathrm{T}(\mathrm{z})$ - as detailed in caption. Numerical simulations with the nonlinear Schrodinger equation (NLSE) of nonlinear beam evolution show a good agreement with IBE predictions. Experimentally, we report the first observation of shifted RBs, obtained by injecting an input Gaussian RB into a 1cm-long cuvette filled with m-cresol/nylon thermal solution as shown in the setup of Fig. 1(c). The experimental results illustrated in Fig. 1(d) are in a good agreement with analytical predictions. Our work open up new possibilities for the control and tailoring of nonlinear beams as well as the study of spatial RWs dynamics in general.
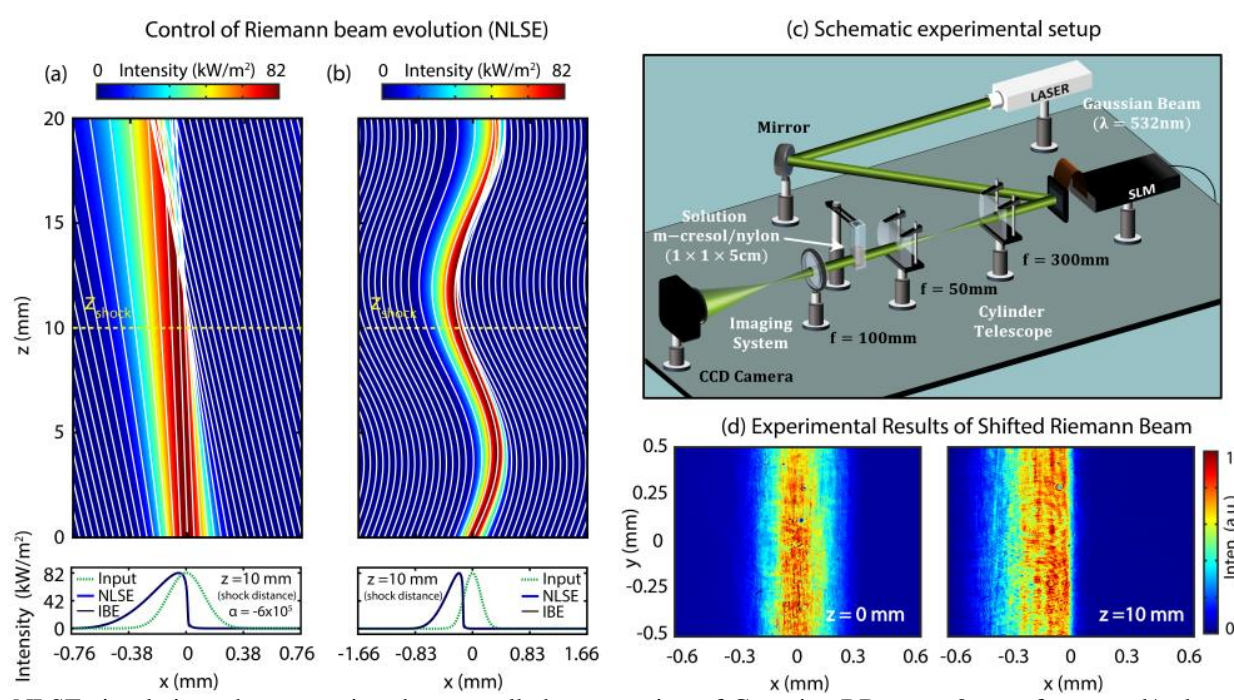

(d) Experimental Results of Shifted Riemann Beam

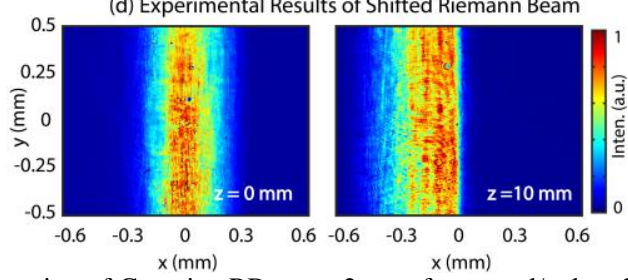

Fig. 1 NLSE simulations demonstrating the controlled propagation of Gaussian RBs over $2 \mathrm{~cm}$ of $\mathrm{m}$-cresol/nylon thermal solution (a) without (shifted RB: $\alpha=-6 \times 10^{5}$ ) and (b) with (sinusoidal RB: T(z) $=3.4 \times 10-{ }^{4} \sin (400 \mathrm{z})$ and $\alpha=-4 \times 10^{5}$ ) the contribution of an external potential. The input RB is tailored to exhibit the formation of a shock formation at $\mathrm{z}=10 \mathrm{~mm}$ (Parameters: Gaussian beam with $82 \mathrm{~W} / \mathrm{m}^{2}$ peak intensity and $240 \mu \mathrm{m}$ waist propagating in an m-cresol/nylon solution: $\mathrm{n}_{0}=1.54, \mathrm{n}_{2}=-1.6 \times 10-5 \mathrm{~W} / \mathrm{cm}^{2}$ at $\left.\lambda=532 \mathrm{~nm}\right)$. Numerical results are compared with the characteristic lines obtained analytically from the IBE (white lines). (c) Experimental setup employed to generate shifted RBs. (d) Transverse intensity patterns showing the formation of the shifted RB in a $10 \mathrm{~mm}$-long nonlinear $\mathrm{m}$-cresol/nylon thermal solution.

\section{References}

[1] J. Bec and K. Khanin, "Burgers Turbulence," Phys. Rep. 447, 1-66 (2007).

[2] B.Wetzel, et al., "Experimental Generation of Riemann Waves in Optics: A Route to Shock Wave Control," Phys. Rev. Lett. 117, 073902 (2016).

[3] S. Randoux, et al., "Optical Random Riemann Waves in Integrable Turbulence," Phys Rev. Lett. 118, 233901 (2017).

[4] W. Wan, S. Jia, and J. W. Fleischer, "Dispersive Superfluid-Like Shock Waves in Nonlinear Optics," Nature Phys. 3, 46 (2007).

[5] S. Trillo, and M. Conforti, "Shock Waves," in G.-D. Peng (Eds.) Handbook of Optical Fibers, pp 1-48, (Springer, Singapore, 2017) 